\title{
Comparative Evaluation of Malaria Rapid Diagnostic Tests and Microscopy
}

\author{
Namita Srivastava $^{1 *}$, Arti Agarwal ${ }^{1}$ and Neeraj Singh ${ }^{2}$ \\ ${ }^{1}$ Department of Microbiology, S.N. Medical College, Agra, U.P., India \\ ${ }^{2}$ Department of Pathology, Vision Pathoplogy, Agra, U.P., India \\ *Corresponding author
}

Malaria is a major public health disease. Over 500 million people are affected worldwide and over one million children are killed annually by malaria (Amazu LU et al., 2009).it is a diagnostic challenge to laboratories in most countries. Most commonly used method for diagnosis is clinical and is the basis for self-treatment. However, the overlapping of malaria symptoms with other tropical diseases makes it unreliable and impairs its specificity. Microscopic examination of Giemsa-stained blood smears has subsequently become the gold standard of malaria diagnosis. Staining techniques have its own set of disadvantages. Poor microscopy has long been attributed to various factors. The list includes training and skills at malaria parasite identification, maintenance, slide preparation techniques, workload, condition of the microscope, and quality of essential laboratory supplies. WHO recommends that malaria case management be based on parasite diagnosis in all cases (World Health Organization 2000)? The use of antigen detecting rapid diagnostic tests (RDTs) is a vital part of this strategy, especially where good-quality microscopy cannot be maintained. RDTs are commercially available in kit form and they are provided with all necessary reagents. They are easy to perform, does not require extensive training or equipments to interpret the results. Results are read in 12-15 min (Moody A, 2002).Thus, they do not require capital investment or electricity, are simple to perform, and are easy to interpret.

The present study was done at Vision pathology Agra over a period of one year in 2016-2017. 1500 cases of suspected malaria fever were enrolled in the study. Malaria smears and rapid diagnostic tests were performed on all samples. Smear microscopy was positive in 1000 cases.500 cases were negative by smear microscopy. RDT was positive in all 1000 cases positive by microscopy. It also detected 50cases which were detected negative by microscopy. The RDT therefore could be said to be as specific as the gold standard i.e. microscopy. Percentage positivity of RDT is $70 \%$ while that of microscopy is $66 \%$. This is in correlation with other studies where RDT was found superior to slide examination (CCA Azikiwe et al., 2012).

This study compared traditional blood smear to rapid antigen capture test thus demonstrates that the RDT, is superior to malaria microscopy for quickly evaluating a patient for malaria. They offer the advantage of more rapid, macroscopic methods for malaria diagnosis, thereby saving on training and 
time. These tests are easy to perform and require little training to interpret the results. Thus, this FDA-approved test appears to be an ideal tool for timely decision making. This is especially true when reliable, experienced microscopy is not immediately available.

\section{References}

Amazu, L.U., Ebong OO, Azikiwe CCA, Unekwe PC, Simialayi MI, Nwosu PJC, et al., Effects of methanolic seeds extract of Carica papaya on Plasmodium berghei infected mice. Asian Pac J Trop Med. 2009; 2(3):1-6.

Azikiwe, C.C.A., CC Ifezulike, IM
Siminialayi, LU Amazu, JC Enye and OE Nwakwunite. A comparative laboratory diagnosis of malaria: microscopy versus rapid diagnostic test kits. Asian Pac J Trop Biomed. 2012 Apr; 2(4): 307-310.

Moody, A., Rapid diagnostic test for malaria parasites. Clin Microbiol Rev. 2002; 15(1):66-78. [PMC free article] [PubMed]

World Health Organization. 2000. WHO/MAL/2000.1091. New perspectives in malaria diagnosis. World Health Organization, Geneva, Switzerland

\section{How to cite this article:}

Namita Srivastava, Arti Agarwal and Neeraj Singh. 2017. Comparative Evaluation of Malaria Rapid Diagnostic Tests and Microscopy. Int.J.Curr.Microbiol.App.Sci. 6(9): 3050-3051. doi: https://doi.org/10.20546/ijcmas.2017.609.375 